The phenotype of mice deficient in PTG suggests that the PTG gene is a candidate gene for type 2 diabetes and insulin resistance in humans. However, previous studies have indicated that PTG gene polymorphism does not contribute to insulin resistance or glucose intolerance $(16,17)$. Given that $\mathrm{G}_{\mathrm{M}}$ gene polymorphism has been associated with insulin resistance in some human populations (18-20), it will be important to reexamine the possible relation between the PTG gene and insulin resistance in humans.

1. Taylor, R., et al. 1996. Direct assessment of liver glycogen storage by ${ }^{13} \mathrm{C}$ nuclear magnetic resonance spectroscopy and regulation of glucose homeostasis after a mixed meal in normal subjects. J. Clin. Invest. 97:126-132.

2. Taylor, R., Price, T.B., Katz, L.D., Shulman, R.G., and Shulman, G.I. 1993. Direct measurement of change in muscle glycogen concentration after a mixed meal in normal subjects. Am. J. Physiol. 265:E224-E229.

3. Magnusson, I., Rothman, D.L., Katz, L.D., Shulman, R.G., and Shulman, G.I. 1992. Increased rate of gluconeogenesis in type II diabetes mellitus. A ${ }^{13} \mathrm{C}$ nuclear magnetic resonance study. J. Clin. Invest. 90:1323-1327.

4. Shulman, G.I., et al. 1990. Quantitation of muscle glycogen synthesis in normal subjects and subjects with non-insulin-dependent diabetes by ${ }^{13} \mathrm{C}$ nuclear magnetic resonance spectroscopy. N. Engl. J. Med. 322:223-228.

5. Cross, D.A.E., Alessi, D.R., Cohen, P., Andjelkovich, M., and Hemmings, B.A. 1995. Inhibition of glycogen synthase kinase- 3 by insulin mediated by protein kinase B. Nature. 378:785-789.

6. Lawrence, J.C., and Roach, P.J. 1997. New insights into the role and mechanism of glycogen synthase activation by insulin. Diabetes. 46:541-547.

7. Printen, J.A., Brady, M.J., and Saltiel, A.R. 1997. PTG, a protein phosphatase 1-binding protein with a role in glycogen metabolism. Science. 275:1475-1478.

8. Crosson, S.M., Khan, A., Printen, J., Pessin, J.E., and Saltiel, A.R. 2003. PTG gene deletion causes impaired glycogen synthesis and developmental insulin resistance. J. Clin. Invest. 111:1423-1432. doi:10.1172/JCI200317975.

9. Suzuki, Y., et al. 2001. Insulin control of glycogen metabolism in knockout mice lacking the muscle-specific protein phosphatase PP1G/ $\mathrm{R}_{\mathrm{GL}}$. Mol. Cell. Biol. 21:2683-2694.

10. Delibegovic, M., et al. 2003. Disruption of the striated muscle glycogen targeting subunit PPP1R3A of protein phosphatase 1 leads to increased weight gain, fat deposition, and development of insulin resistance. Diabetes. 52:596-604.

11. Ryysy, L., et al. 2000. Hepatic fat content and insulin action on free fatty acids and glucose metabolism rather than insulin absorption are associated with insulin requirements during insulin therapy in type 2 diabetic patients. Diabetes. 49:749-758.

12. Jacob, S., et al. 1999. Association of increased intramyocellular lipid content with insulin resistance in lean nondiabetic offspring of type 2 diabetic subjects. Diabetes. 48:1113-1119.

13. Kim, J.K., et al. 2001. Tissue-specific overexpres- sion of lipoprotein lipase causes tissue-specific insulin resistance. Proc. Natl. Acad. Sci. U. S. A 98:7522-7527.

14. Godbole, V., and York, D.A. 1978. Lipogenesis in situ in the genetically obese Zucker fatty rat $(\mathrm{fa} / \mathrm{fa})$ : role of hyperphagia and hyperinsulinaemia. Diabetologia. 14:191-197.

15. Hribal, M.L., Oriente, F., and Accili, D. 2002 Mouse models of insulin resistance. Am. J. Physiol. Endocrinol. Metab. 282:E977-E981.

16. Permana, P.A., Luczy-Bachman, G., and Bogardus, C. 1999. Protein targeting to glycogen/PPP1R5: screening of coding and flanking genomic regions for polymorphisms and association analysis with insulin action in Pima Indians. Biochem. Biophys. Res. Commun. 258:184-186.

17. Hansen, L., et al. 1999. Mutational analysis of the coding regions of the genes encoding protein kinase $B-\alpha$ and $-\beta$, phosphoinositide-dependent protein kinase-1, phosphatase targeting to glycogen, protein phosphatase inhibitor-1, and glycogenin - lessons from a search for genetic vari ability of the insulin-stimulated glycogen synthesis pathway of skeletal muscle in NIDDM patients. Diabetes. 48:403-407.

18. Hansen, L., et al. 1995. A widespread amino acid polymorphism at codon 905 of the glycogenassociated regulatory subunit of protein phosphatase- 1 is associated with insulin resistance and hypersecretion of insulin. Hum. Mol. Genet. 4:1313-1320.

19. Xia, J., et al. 1998. A common variant in $P P P 1 R 3$ associated with insulin resistance and type 2 diabetes. Diabetes. 47:1519-1524

20. Maegawa, H., et al. 1999. The 3'-untranslated region polymorphism of the gene for skeletal muscle-specific glycogen-targeting subunit of protein phosphatase 1 in the type 2 diabetic Japanese population. Diabetes. 48:1469-1472.

\title{
Connecting the dots from Toll-like receptors to innate immune cells and inflammatory bowel disease
}

\section{David L. Boone and Averil Ma}

Department of Medicine, Ben May Institute for Cancer Research, The University of Chicago, Chicago, Illinois, USA

J. Clin. Invest. 111:1284-1286 (2003). doi:10.1172/JCI200318545.

Address correspondence to: Averil Ma, University of Chicago Hospitals MC 6084, Department of Medicine, 5841 S. Maryland Avenue, Chicago, Illinois 60637, USA. Phone: (773) 834-0687; Fax: (773) 702-2281 E-mail: ama@medicine.bsd.uchicago.edu. Conflict of interest: The authors have declared that no conflict of interest exists. Nonstandard abbreviations used: inflammatory bowel disease (IBD); CTL antigen-4 (CTLA-4); suppressor of cytokine signaling (SOCS); Src homology protein-1 (SHP-1); IL-10 receptor (IL-10R); Toll-like receptor (TLR); pathogen-associated molecular product (PAMP).
The etiologies of inflammatory bowel diseases (IBD) are not known but are thought to involve a genetic predisposition toward exaggerated inflammatory responses to enteric flora. Effective treatments for IBD are therefore predicated on the regulation of inflammatory responses in the intestine. Most current therapeutic agents for IBD, including 5-ASA, prednisone, and anti-TNF antibody are directed at the reduction of proinflammatory molecules. Recently, a number of negative regulatory molecules (e.g., IL-10, TGF- $\beta$, CTL antigen-4 [CTLA-4], Fas, suppressor of cytokine signaling [SOCS] proteins, A20, and Src homology protein-1 [SHP-1]), which either bind to effector immune cells and inhibit their activation (e.g., IL-10, TGF- $\beta$, and CTLA-4), induce programmed cell death (e.g., Fas), or regulate intracellular signaling pathways (e.g., SOCS proteins, SHP-1, and A20), have been identified. These negative regulatory molecules may provide novel therapeutic targets for the treatment of IBD.

\section{IL-10, Stat3, and IBD}

Among these negative regulators of inflammation, IL-10 inhibits multiple cell types, including macrophages (1, 2). The physiological importance of IL-10 is highlighted by the spontaneous development of bowel inflammation in IL-10-deficient $\left(I L-10^{-/-}\right)$ mice $(3,4)$. The inflamed mucosa of $I L-10^{-/-}$mice contains elevated num- 


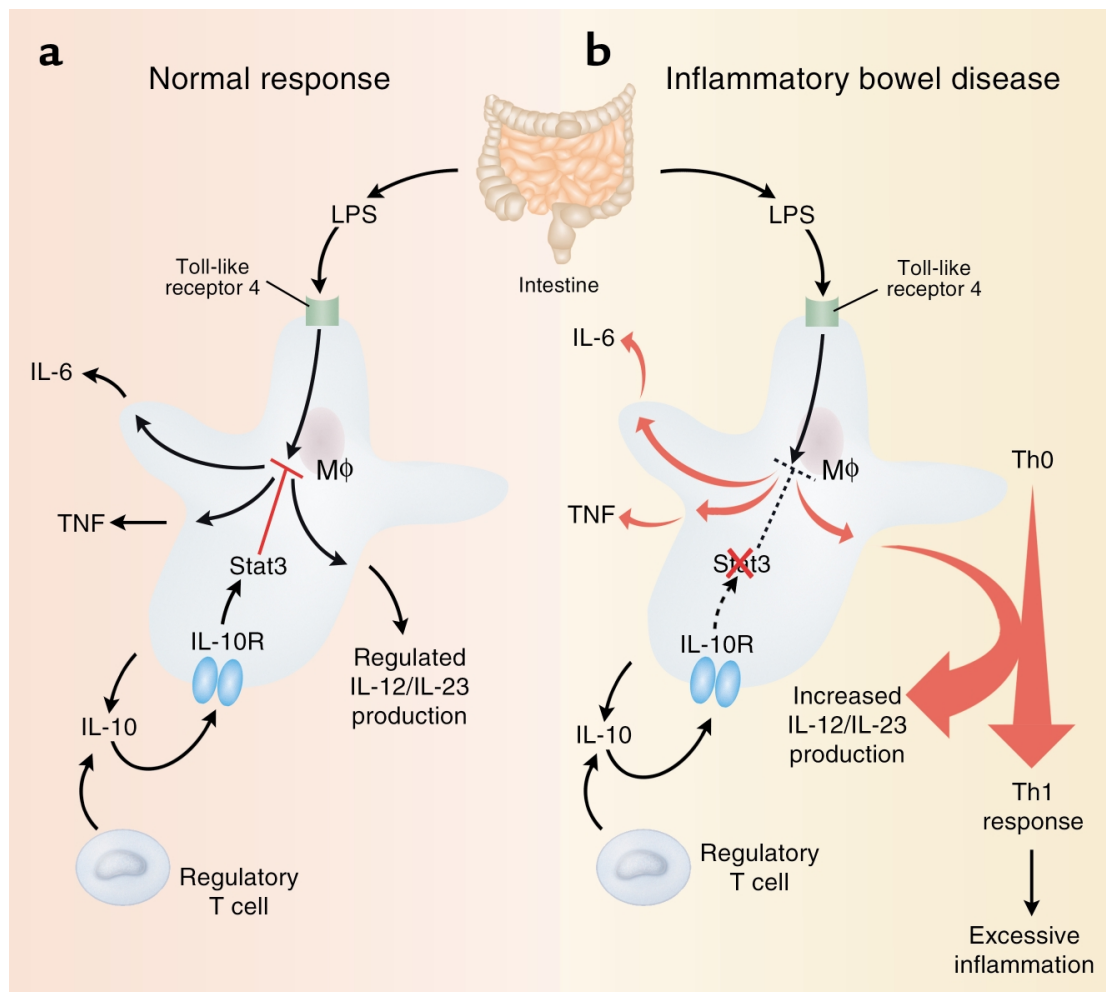

Figure 1

Model illustrating the roles of the Toll-like receptor (TLR) and Stat3 in IBD. Myeloid cells respond to LPS-mediated TLR stimulation. Normally, inflammation is controlled by IL-10, which stimulates myeloid cell Stat3 activation to suppress TLR-induced IL-12/IL-23 production (a). Kobayashi et al. (7) demonstrate that in LysMCre/Stat3flox- mice, IL-10 does not effectively suppress IL-12/IL-23 production. IL-12/IL-23 release activates lymphocytes, causing an exaggerated bias toward Th1-type inflammation (b).

bers of myeloid cells, IFN- $\gamma$-producing $\mathrm{CD} 4^{+} \mathrm{T}$ cells, and high levels of proinflammatory cytokines such as IL-1, IL-12, IL-6, and TNF (4). Antibodyinduced depletion of IFN- $\gamma$ or IL-12 abrogates or prevents, respectively, spontaneous inflammation in $I L-10^{-/-}$ mice (4). Furthermore, $R A G-2^{-/-}$mice reconstituted with $I L-10^{-/-} \mathrm{CD} 4^{+} \mathrm{T}$ cells develop bowel inflammation while

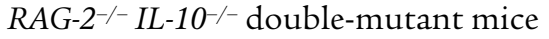
do not. Therefore, Th1 biased $\mathrm{CD} 4^{+} \mathrm{T}$ cells appear to be pathogenic in $I L-10^{-1}$ mice (4). Finally, $I L-10^{-/-}$mice do not develop disease when raised in gnotobiotic conditions but do develop IBD when transferred to conventional facilities, indicating a role for enteric flora in the pathology of this IBD model (4, $5)$. Taken together, these data suggest that $\mathrm{CD}^{+} \mathrm{T}$ cell production of IL-10 prevents the development of a $\mathrm{CD} 4^{+} \mathrm{T}$ cell-mediated, IL-12-driven, Th1-type inflammation in the intestine that is initiated by the presence of microbes in the gut lumen.
The mechanism(s) by which IL-10 signals inhibit immune cell activation is poorly understood. IL-10 binds to a recently described IL-10 receptor (IL-10R) complex that is composed of at least two subunits, IL-10R $\alpha$ and IL-10R $\beta$ - also known as CRFB4 or CRF2-4 (2). IL-10R signaling in macrophages requires the kinase Jak-1 and the transcription factor Stat3 (2). The essential role of Stat 3 in mediating IL-10 signals in myeloid cells was demonstrated by the targeted deletion of Stat 3 in myeloid cells (LysMCre/Stat $3^{\text {flox- }}$ mice) that rendered neutrophils and macrophages unresponsive to IL-10 (6). This myeloid unresponsiveness to IL-10 resulted in the development of a polarized Th1-type immune response and chronic enterocolitis (6). Thus, the specific inability of myeloid cells to respond to IL-10 through Stat3 signals appears to recapitulate spontaneous inflammation seen in $I L-10^{-/-}$mice.

\section{Cytokines and IBD in myeloid}

\section{Stat3-deficient mice}

In this issue of the JCI Kobayashi et al. (7) perform multiple genetic manipulations of the LysMCre/Stat $3^{f l o x-}$ mouse to elucidate the sequential innate and adaptive immune processes that lead to the development of this Th1-type IBD. First, they address the questions of whether IFN- $\beta$, TNF, or IL-12p40 contribute to the enterocolitis and Th1 profile of LysMCre/Stat 3 flox- mice by interbreeding those mice to Stat1 $1^{-/-}$, $T N^{-1-}$, and $I L-12 p 40^{-/-}$mice, respectively. These experiments show that Stat 1 and TNF are dispensable, whereas IL-12p40 is required for enhanced Th1 responses and enterocolitis in LysMCre/Stat $3^{\text {flox- }}$ mice. The deletion of IL-12p40 ablates both IL-12 and IL-23, a proinflammatory cytokine that shares the IL-12p40 subunit $(8,9)$, so it is not clear whether the lack of IL-12, IL-23, or both results in the amelioration of enterocolitis in $I L-12 p 40^{-/-} \times$ LysMCre/Stat3flox- mice. Kobayashi et al. also find that disease is prevented in RAG-2-/- $\times$ LysMCre/Stat3flox- mice, despite high levels of IL-12p40, demonstrating a requirement for adaptive lymphocytes in this model of IBD.

\section{LPS, TLR4, and IBD in myeloid \\ Stat3-deficient mice}

The first and perhaps critical step in initiating immune responses is typically the engagement of host Toll-like receptor (TLR) molecules by conserved pathogen associated molecular products (PAMPs) (10). TLR ligation by PAMPs induces the activation of NF- $\mathrm{KB}$ and other transcription factors resulting in the production of multiple proinflammatory molecules. Recently, it has been appreciated that TLRs also influence the nature of the immune response, in particular $\mathrm{T}$ cell skewing toward a Th1 or Th2 profile. Myeloid cells, which are exquisitely sensitive to TLR ligands and produce significant IL-12p 40, are therefore poised to play key roles in the initiation and possibly the Th1/Th2 skewing of inflammatory responses. The potency of myeloid cell TLR responses also warrants their effective negative regulation to prevent pathological inflammation. Kobayashi et al. (7) address the potential role of TLRs in the IL-12-driven IBD of 
LysMCre/Stat $3^{\text {flox- }}$ mice by interbreeding with $T L R 4^{-1-}$ mice. They show that TLR $4^{-/-} \times$LysMCre/Stat $3{ }^{\text {flox- }}$ mice display dramatically reduced intestinal inflammation compared to LysMCre/Stat $3^{\text {flox- }}$ mice. This disease amelioration is consistent with the requirement for microbe or microbial products in the pathology of IBD in $I L-10^{-/-}$mice $(4,5)$.

\section{A model for the role of IL-10, TLR4, and Stat3 in IBD}

This work suggests a potential model (Figure 1) for the sequential activation and negative regulation of innate and adaptive immune cells during intestinal inflammation: LPS, perhaps from the intestinal lumen, induces the production of IL-12/IL-23 by myeloid cells, which drives a Th1-type inflammatory process in the lymphocyte population. The model suggests that inflammation would normally be controlled by myeloid or lymphocytederived IL-10 acting through Stat 3 in myeloid cells to block further production of IL-12/IL-23. This suppressive IL-10 signal may be particularly important in the gut, where immune cells are continuously in close contact with LPS and other microbial PAMPs. In LysMCre/ Stat $3^{\text {flox- }}$ mice, IL-10 does not effectively suppress IL-12/IL-23 production by myeloid cells, resulting in the expansion and Th 1 bias of CD4 ${ }^{+} \mathrm{T}$ cells. This study by Kobayashi et al. (7) clearly places TLR-induced myeloid IL-12/ IL-23 production as an important target of IL-10-induced, Stat3-mediated anti-inflammatory effects. Additionally, this study points to important unanswered questions regarding the anti-inflammatory effects of IL-10 including: How is IL-10 receptor expression regulated in myeloid cells? How does IL-10 activation of Stat 3 regulate TLR-induced expression of IL-12p40?

The experimental evidence above and other data suggest that IL-10 might be a promising target for alleviating inflammation in human patients with IBD. Specifically, the evidence for the potential therapeutic use of IL-10 includes the fact that IL-10 suppresses the production of inflammatory cytokines by multiple immune cell types; continuous IL-10 treatment can prevent or ameliorate disease in several mouse models of IBD; $I L-10^{-/-}$ mice develop IBD; and small trials of treatment with IL-10 in patients with ulcerative colitis showed reduced cytokine levels and improved histological scores $(4,11)$. It is therefore vexing that recent clinical trials found no beneficial effect of IL-10 in the treatment of Crohn disease $(12,13)$. It may be that the nature of the IBD being treated, the timing of IL-10 administration, the route of drug delivery, or the specific cell types being targeted by IL-10 will affect the outcome of the therapy (14). Additionally, IL-10 may be more effective when used in consort with other novel or established treatments for IBD. It is clear that further experimental dissection, like that performed by Kobayashi et al. (7), of the cell types and/or factors that mediate the antiinflammatory effects of IL-10 in the intestinal mucosa are warranted.

1. Fiorentino, D.F., Bond, M.W., and Mosmann, T.R. 1989. Two types of mouse T helper cell. IV. Th2 clones secrete a factor that inhibits cytokine pro- duction by Th1 clones. J. Exp. Med 170:2081-2095

2. Moore, K.W., de Waal Malefyt, R., Coffman, R.L., and O'Garra, A. 2001. Interleukin-10 and the interleukin-10 receptor. Annu. Rev. Immunol. 19:683-765.

3. Kuhn, R., Lohler, J., Rennick, D., Rajewsky, K., and Muller, W. 1993. Interleukin-10-deficient mice develop chronic enterocolitis. Cell. 75:263-274.

4. Rennick, D.M., and Fort, M.M. 2000. Lessons from genetically engineered animal models. XII IL-10-deficient (IL-10-/-) mice and intestinal inflammation. Am. J. Physiol. Gastrointest. Liver Physiol. 278:G829-G833.

5. Sellon, R.K., et al. 1998. Resident enteric bacteria are necessary for development of spontaneous colitis and immune system activation in interleukin-10-deficient mice. Infect. Immun. 66:5224-5231.

6. Takeda, K., et al. 1999. Enhanced Th1 activity and development of chronic enterocolitis in mice devoid of Stat 3 in macrophages and neutrophils. Immunity. 10:39-49.

7. Kobayashi, M., et al. 2003. Toll-like receptordependent production of IL-12p40 causes chronic enterocolitis in myeloid cell-specific Stat3-deficient mice. J. Clin. Invest. 111:1297-1308. doi:10.1172/JCI200317085.

8. Oppmann, B., et al. 2000. Novel p19 protein engages IL-12p40 to form a cytokine, IL-23, with biological activities similar as well as distinct from IL-12. Immunity. 13:715-725.

9. Cua, D.J., et al. 2003. Interleukin-23 rather than interleukin-12 is the critical cytokine for autoimmune inflammation of the brain. Nature. 421:744-748.

10. Janeway, C.A., Jr., and Medzhitov, R. 2002. Innate immune recognition. Annu. Rev. Immunol. 20:197-216.

11. van Deventer, S.J., Elson, C.O., and Fedorak, R.N 1997. Multiple doses of intravenous interleukin 10 in steroid-refractory Crohn's disease. Crohn's Disease Study Group. Gastroenterology. 113:383-389.

12. Fedorak, R.N., et al. 2000. Recombinant human interleukin 10 in the treatment of patients with mild to moderately active Crohn's disease. The Interleukin 10 Inflammatory Bowel Disease Cooperative Study Group. Gastroenterology. 119:1473-1482.

13. Schreiber, S., et al. 2000. Safety and efficacy of recombinant human interleukin 10 in chronic active Crohn's disease. Crohn's Disease IL-10 Cooperative Study Group. Gastroenterology. 119:1461-1472.

14. Madsen, K. 2002. Combining T cells and IL-10: a new therapy for Crohn's disease? Gastroenterology. 123:2140-2144. 